FERMILAB-Pub-96/122-A

astro-ph/9606059

submitted to Physical Review Letters

\title{
The Big-Bang Nucleosynthesis Limit to the Number of Neutrino Species
}

\author{
Craig J. Copi, ${ }^{1,2}$ David N. Schramm, ${ }^{1,2,3}$ and Michael S. Turner ${ }^{1,2,3}$ \\ ${ }^{1}$ Department of Physics \\ Enrico Fermi Institute, The University of Chicago, Chicago, IL 60637-1433 \\ ${ }^{2}$ NASA/Fermilab Astrophysics Center \\ Fermi National Accelerator Laboratory, Batavia, IL 60510-0500 \\ ${ }^{3}$ Department of Astronomy \& Astrophysics \\ The University of Chicago, Chicago, IL 60637-1433
}

\begin{abstract}
Concern about systematic uncertainty in the ${ }^{4} \mathrm{He}$ abundance as well as the chemical evolution of ${ }^{3} \mathrm{He}$ leads us to re-examine this important limit. It is shown that with conservative assumptions no more than the equivalent of 4 massless neutrino species are allowed. Even with the most extreme estimates of the astrophysical uncertainties a meaningful limit still exists, less than 5 massless neutrino species, and illustrates the robustness of this argument. A definitive measurement of the deuterium abundance in high-redshift hydrogen clouds should soon sharpen the limit.
\end{abstract}


Introduction. Big-bang nucleosynthesis is one of the experimental pillars of the standard cosmology [1, 2]. It also probes particle physics. Among other things, it has been used to set a stringent limit to the energy density contributed by light (mass $\ll \mathrm{MeV}$ ) particle species, usually quantified as the equivalent number of massless neutrino species $\left(\equiv N_{\nu}\right)$ [3]. This limit indicated that the number of neutrino species was small before accelerator experiments were able to experimentally establish this directly, and further, has served to constraint theories proposed to unify the forces and particles of Nature. Since the cosmological bound also constrains light species that do not couple to the $Z^{0}$, it is an important complement to the LEP measurement, $N_{\nu}=2.991 \pm 0.016$.

The physics underlying the neutrino limit is simple: the big-bang production of ${ }^{4} \mathrm{He}$ increases with both baryon density (quantified by the present baryon to photon ratio $\eta$ ) and the number of massless neutrino species. Thus, an upper limit to the primeval ${ }^{4} \mathrm{He}$ abundance $\left(\equiv Y_{P}\right)$ and a lower limit to the baryon density lead to an upper limit to $N_{\nu}$. The lower limit to the baryon density is based upon the big-bang production of deuterium, which rises rapidly with decreasing baryon density 4 . Because D is easily destroyed in stars, first being burnt to ${ }^{3} \mathrm{He}$, the limit actually hinges upon $\mathrm{D}+{ }^{3} \mathrm{He}$, bringing in the chemical evolution of ${ }^{3} \mathrm{He}$. This has been the standard approach for setting a limit to $N_{\nu}$.

Over the past five years limits to $N_{\nu}$ ranging from 3.04 to around 5 have been quoted [5]. The disparity arises because the irreducible uncertainties are systematic, rather than statistical. In particular, the pressing issues involve the primeval abundance of ${ }^{4} \mathrm{He}$ and the chemical evolution of ${ }^{3} \mathrm{He}$ (astronomers use the term "chemical evolution" to refer to nuclear processing). The purpose of this paper is to clarify the current situation, to use a new technique to obtain a limit to $N_{\nu}$ that is independent of the chemical evolution of ${ }^{3} \mathrm{He}$, and to show how measurements of the primeval D abundance in high-redshift hydrogen clouds should soon sharpen the neutrino limit. In so doing, we will emphasize the robustness of the cosmological limit to $N_{\nu}$.

The light-element abundances. To orient the reader we begin with a brief overview. The big-bang production of $\mathrm{D},{ }^{3} \mathrm{He},{ }^{4} \mathrm{He}$ and ${ }^{7} \mathrm{Li}$ is summarized in Fig. 1. The predicted and measured abundances are consistent - within the uncertainties - for $\eta \simeq(2-7) \times 10^{-10}$ [1, 6]. The status of the four light elements is as follows.

1. The lithium abundance is measured in the atmospheres of the oldest stars in our galaxy (pop II halo stars) and is relatively well determined, $\left({ }^{7} \mathrm{Li} / \mathrm{H}\right)_{\text {pop II }}=(1.4 \pm 0.3) \times 10^{-10}$ [1], 7]. Because ${ }^{7} \mathrm{Li}$ burns at a low temperature, the most important concern is that ${ }^{7} \mathrm{Li}$ may have been depleted. Stellar models indicate that a depletion of up to a factor of about two is consistent with the observations of ${ }^{7} \mathrm{Li}$ and other light elements in these old stars [8].

2. The ${ }^{4} \mathrm{He}$ abundance increases with time, as ${ }^{4} \mathrm{He}$ is made by stars, and so any measurement provides an upper bound to the primeval component. The primeval abundance is extrapolated from measurements of metal-poor, extragalactic HII regions (hot clouds of ionized $\mathrm{H}$ and $\mathrm{He}$ gas). A compilation and analysis of the extant data gave 
$Y_{P}=0.232 \pm 0.003$ (stat) \pm 0.005 (sys) [9]. Others have estimated the systematic uncertainty to be larger, by a factor of two or even more [10]; it arises from many sources, from uncertainties in theoretical emissivities to assumptions and approximations made in modeling the HII regions. A very recent study of 27 new extragalactic HII regions, using a new set of emissivities, finds $Y_{P}=0.243 \pm 0.003$ [1].

3. Deuterium is destroyed in the contemporary Universe, being burnt to ${ }^{3} \mathrm{He}$, which links its post-big-bang history to that of ${ }^{3} \mathrm{He}$. The deuterium abundance has been measured in the local interstellar medium (ISM) and in the pre-solar nebula [12]. While neither value is expected to reflect the primeval abundance, both place a lower limit to the big-bang production and thereby a firm upper limit to the baryon density. This twodecade old limit, $\Omega_{B} \lesssim 0.06\left(70 \mathrm{~km} \mathrm{sec}^{-1} \mathrm{Mpc}^{-1} / H_{0}\right)^{2}$, precludes baryons from closing the Universe [13], and is not being questioned.

The primeval deuterium abundance can be determined by measuring the deuterium Ly- $\alpha$ absorption feature in high-redshift $(z \gtrsim 2)$ hydrogen clouds which are backlit by distant QSOs [14, 15]. There are now three claimed detections [16] and four tentative detections [17]; the reported values are from $(\mathrm{D} / \mathrm{H})_{P} \simeq 2 \times 10^{-5}$ to $2 \times 10^{-4}$, spanning the range anticipated on the basis of the big-bang production of the other light-elements. A definitive determination will peg the baryon-to-photon ratio to an accuracy of around $10 \%$ because of the rapid variation of $\mathrm{D}$ production with $\eta$.

4. Since all measurements of ${ }^{3} \mathrm{He}$ involve samples that have to some extent been processed through stars, chemical evolution is the central issue. The chemical evolution of ${ }^{3} \mathrm{He}$ is complicated and not well understood. Stars burn D to ${ }^{3} \mathrm{He}$ before reaching the main sequence; this is not controversial. According to conventional stellar models, massive stars $\left(M \gtrsim 8 M_{\odot}\right)$ reduce the ${ }^{3} \mathrm{He}$ in material they return to the ISM, by a factor of around five or so. Since high-mass stars also produce elements heavier than ${ }^{4} \mathrm{He}$ ("metals"), there is a limit to the amount of processing through high-mass stars - and thus to ${ }^{3} \mathrm{He}$ destruction. Low-mass stars $\left(M \lesssim 2 M_{\odot}\right)$ preserve and add significant ${ }^{3} \mathrm{He}$ to the material they return to the ISM [18]. This leads to the conclusion that the sum of $\mathrm{D}+{ }^{3} \mathrm{He}$ should have decreased by at most a modest factor since primordial nucleosynthesis, which has been used to obtain the lower bound to $\eta$ needed to constrain the number of massless neutrino species $[4$.

There is little empirical evidence for the conventional view concerning the chemical evolution of ${ }^{3} \mathrm{He}$; moreover, it has recently been challenged both theoretically and observationally. Slow-mixing mechanisms that would transport ${ }^{3} \mathrm{He}$ deep enough to be burnt have been suggested to explain oxygen and carbon isotopic ratios measured in some stars and meteorites [19]. In addition, even if the conventional wisdom is correct in the mean, galactic chemical abundances are heterogeneous and the $\mathrm{D}+{ }^{3} \mathrm{He}$ abundance has only been measured locally [20].

On the observational side, a recent measurement of the ${ }^{3} \mathrm{He}$ abundance in the ISM indicates that the sum of $\mathrm{D}+{ }^{3} \mathrm{He}$ has not increased in the past 4.5 Gyr [21, 22]; 
conventional models predict an increase since the recent evolution of the ISM should be dominated by low-mass stars. Finally, if the highest values of the D abundance measured in high-redshift hydrogen clouds are correct, then the present $\mathrm{D}+{ }^{3} \mathrm{He}$ abundance is too small by a factor of almost ten to accommodate conventional models.

A new approach. The two major obstacles to setting a reliable limit to $N_{\nu}$ are the chemical evolution of ${ }^{3} \mathrm{He}$ - which affects the lower bound to $\eta$ based upon $\mathrm{D}+{ }^{3} \mathrm{He}-$ and the systematic uncertainty in the primeval ${ }^{4} \mathrm{He}$ - which affects the upper bound to big-bang ${ }^{4} \mathrm{He}$ production. To clarify and to minimize the dependence upon these we have computed limits to $N_{\nu}$ based upon a likelihood function for $\tilde{N} \equiv N_{\nu}-\Delta Y / 0.016$ which uses the ${ }^{7} \mathrm{Li}$ and ${ }^{4} \mathrm{He}$ abundances 7 and takes deuterium into account only by means of a Bayesian prior. This approach exploits the fact that ${ }^{7} \mathrm{Li}$ increases with $N_{\nu}$ rapidly enough to provide a meaningful lower limit to $\eta$ as well as the fact that $N_{\nu}$ and a systematic shift $(\Delta Y)$ in the assumed primeval ${ }^{4} \mathrm{He}$ abundance are equivalent (see Ref. [6]).

In computing $\mathcal{L}(\tilde{N})$ we take $Y_{P}=0.242+\Delta Y \pm 0.003$ and consider two possibilities for ${ }^{7} \mathrm{Li},\left({ }^{7} \mathrm{Li} / \mathrm{H}\right)_{P}=(1.4 \pm 0.3) \times 10^{-10}$ (no depletion) and $(3 \pm 0.6) \times 10^{-10}$ (factor of two depletion), using whichever gives the less stringent limit to $N_{\nu}$. Regarding the primeval ${ }^{4} \mathrm{He}$ abundance; as a "standard case" we take the central value $Y_{P}=0.242,0.01$ larger than the central value recommended in Ref. [9] - essentially equal to the value derived from the newest data set [11] - and roughly equal to the upper limit to $Y_{P}$ used previously in deriving a limit to $N_{\nu}$. Through $\Delta Y$ we allow for possible systematic error in the ${ }^{4} \mathrm{He}$ abundance and explore the sensitivity of the neutrino limit to it.

We consider four priors for deuterium: $(1)(\mathrm{D} / \mathrm{H})_{P} \leq 1.0$, corresponding to essentially no prior information; $(2)(\mathrm{D} / \mathrm{H})_{P} \leq 2 \times 10^{-4}$, corresponding to the previous bound based upon $\mathrm{D}+{ }^{3} \mathrm{He}$ 四; $(3)$ a distribution for $(\mathrm{D} / \mathrm{H})_{P}$ based upon an extreme chemical evolution model [22], which allows for destruction of ${ }^{3} \mathrm{He}$ in low-mass stars and extra destruction in high-mass stars by assuming that metals they produce were ejected into the intergalactic medium, avoiding the Galactic metallicity constraint; and $(4)(\mathrm{D} / \mathrm{H})_{P}=(2.5 \pm 0.75) \times 10^{-5}$, an example of the accuracy to which the primeval deuterium might be determined. Our four likelihood functions are shown in Fig. 2.

Discussion. Credibility intervals for $N_{\nu}$ are obtained from $\mathcal{L}(\tilde{N})$ by choosing a value for $\Delta Y$ and imposing a prior on $N_{\nu}$, either $N_{\nu} \geq 3$ or $N_{\nu} \geq 2$. As Table 1 illustrates, better knowledge of the deuterium abundance and/or a tighter limit to the primeval ${ }^{4} \mathrm{He}$ abundance improve the limit to $N_{\nu}$.

Because our likelihood is a function of $\tilde{N}=N_{\nu}-\Delta Y / 0.016$, in the absence of a prior for $N_{\nu}$ the limit to $N_{\nu}$ would scale precisely as the limit to $\tilde{N}$ plus $\Delta Y / 0.016$; e.g., increasing $Y_{P}$ by 0.01 would raise the limit by about 0.6 neutrino species. This scaling works reasonably well for the $N_{\nu} \geq 2$ prior.

Regarding the dependence of the neutrino limit upon deuterium:

\footnotetext{
${ }^{1}$ The authors of Ref. [23] have advocated the use of ${ }^{7} \mathrm{Li}$ and ${ }^{4} \mathrm{He}$ alone, albeit in a different context.
} 


\begin{tabular}{cccc}
\hline $\mathrm{D} / \mathrm{H}\left({ }^{7} \mathrm{Li}\right)$ & $Y_{P}$ & $N_{\nu} \geq 2$ & $N_{\nu} \geq 3$ \\
\hline \hline \multirow{3}{*}{1.0 (high) } & $0.232 \pm 0.003$ & $2.0-2.6,2.8-3.6$ & $3.0-3.7$ \\
& $0.237 \pm 0.003$ & $2.0-2.9,3.2-3.9$ & $3.0-4.0$ \\
& $0.242 \pm 0.003$ & $\mathbf{2 . 3 - 3 . 3 , 3 . 4 - 4 . 3}$ & $\mathbf{3 . 0 - 3 . 3 , ~ 3 . 4 - 4 . 3}$ \\
& $0.247 \pm 0.003$ & $2.6-3.6,3.8-4.6$ & $3.0-3.6,3.7-4.6$ \\
& $0.252 \pm 0.003$ & $2.9-3.9,4.1-4.9$ & $3.0-3.9,4.1-4.9$ \\
\hline \multirow{3}{*}{$2 \times 10^{-4}$ (low) } & $0.232 \pm 0.003$ & $2.0-3.1$ & $3.0-3.3$ \\
& $0.237 \pm 0.003$ & $2.3-3.4$ & $3.0-3.5$ \\
& $0.242 \pm 0.003$ & $\mathbf{2 . 6 - 3 . 7}$ & $\mathbf{3 . 0 - 3 . 7}$ \\
& $0.247 \pm 0.003$ & $2.9-4.1$ & $3.0-4.0$ \\
& $0.252 \pm 0.003$ & $3.2-4.4$ & $3.2-4.4$ \\
\hline \multirow{2}{*}{ extreme chemical } & $0.232 \pm 0.003$ & $2.0-2.6$ & $3.0-3.5$ \\
evolution for ${ }^{3} \mathrm{He}($ high $)$ & $0.237 \pm 0.003$ & $2.1-2.9$ & $3.0-3.6$ \\
& $0.242 \pm 0.003$ & $\mathbf{2 . 4 - 3 . 2}$ & $\mathbf{3 . 0 - 3 . 4}$ \\
& $0.247 \pm 0.003$ & $2.6-3.7$ & $3.0-3.6$ \\
& $0.252 \pm 0.003$ & $2.9-4.0$ & $3.0-3.8$ \\
\hline & $0.232 \pm 0.003$ & $2.0-2.4$ & $3.0-3.1$ \\
& $0.237 \pm 0.003$ & $2.0-2.7$ & $3.0-3.2$ \\
$(2.5 \pm 0.75) \times 10^{-5}$ (high) & $0.242 \pm 0.003$ & $\mathbf{2 . 3 - 3 . 0}$ & $\mathbf{3 . 0 - 3 . 2}$ \\
& $0.247 \pm 0.003$ & $2.6-3.3$ & $3.0-3.4$ \\
& $0.252 \pm 0.003$ & $2.9-3.6$ & $3.0-3.6$ \\
\hline \hline
\end{tabular}

Table 1: The 95\% credible intervals for $N_{\nu}$ based upon the different deuterium and $N_{\nu}$ priors and central values of the primeval ${ }^{4} \mathrm{He}$ abundance. High (low) indicates high (low) ${ }^{7} \mathrm{Li}$ abundance was used to derive the limit (see text). 
1. Using the original $\mathrm{D}+{ }^{3} \mathrm{He}$ bound, $(\mathrm{D} / \mathrm{H})_{P}<2 \times 10^{-4}$ 国, the $95 \%$ credible region extends to $\tilde{N}=3.3$. For $Y_{P}=0.242, N_{\nu}<3.7(3.7)$ and for $Y_{P}=0.252, N_{\nu}<4.4(4.4)$. (The numbers in parentheses are for the prior $N_{\nu} \geq 2$.)

2. Using the probability distribution for deuterium based an extreme model for the chemical evolution of ${ }^{3} \mathrm{He}$, the $95 \%$ credible region only extends to $\tilde{N}=3.2$. For $Y_{P}=0.242$, $N_{\nu}<3.4(3.2)$ and for $Y_{P}=0.252, N_{\nu}<3.8(4.0)$. The use of a probability distribution for $(\mathrm{D} / \mathrm{H})_{P}$ actually improves the bound slightly, in spite of the extreme model for chemical evolution assumed.

3. If $(\mathrm{D} / \mathrm{H})_{P}$ is determined to be $(2.5 \pm 0.75) \times 10^{-5}$ from high-redshift hydrogen clouds, the $95 \%$ credible limit region extends to $\tilde{N}=3.0$. For $Y_{P}=0.242, N_{\nu}<3.2(3.0)$ and for $Y_{P}=0.252, N_{\nu}<3.6(3.6)$.

4. With no information about deuterium, $(\mathrm{D} / \mathrm{H})_{P}<1.0$, the likelihood function for $\tilde{N}$ is double-peaked. The two peaks correspond to two ways of accommodating both ${ }^{4} \mathrm{He}$ and ${ }^{7} \mathrm{Li}$ : low $\tilde{N}$ and high $\eta$ and high $\tilde{N}$ and low $\eta$. The upper boundary of the $95 \%$ credible region is $\tilde{N}=4.3$. For a central value $Y_{P}=0.242, N_{\nu}<4.3(4.3)$ and for $Y_{P}=0.252$ (four times the systematic error estimated in Ref. [9]), $N_{\nu}<4.9(4.9)$.

Several points should be noted. (1) Because ${ }^{7} \mathrm{Li}$ depletion in pop II halo stars cannot be proved nor disproved at the moment, our limits are based upon the ${ }^{7} \mathrm{Li}$ abundance that gives the less stringent limit. Once the issue of depletion is resolved, the limits may improve by a modest amount. Our limits are relatively insensitive to even larger Li depletion. For example going from a factor of two depletion to a factor of three depletion changes the neutrino limit by at most 0.2. (2) If the primeval deuterium abundance is determined to be at the high end of the current range, say $(\mathrm{D} / \mathrm{H})_{P}=(1.0 \pm 0.3) \times 10^{-4}$, the limits are essentially as in case (1) above. This is because such a measurement would pin $\eta$ at very nearly the same value as the $\mathrm{D}+{ }^{3} \mathrm{He}$ bound. However, should $(\mathrm{D} / \mathrm{H})_{P} \simeq(2 \pm 0.6) \times 10^{-4}$, the limit worsens slightly (to 4.3 for a central $Y_{P}=0.242$ ), because $\eta$ is pinned to a lower value. (3) The limits quoted here are not directly comparable to other work. The most similar approach is that of Fields et al. [5]; however, they determine a lower limit to $\eta$ based upon ${ }^{4} \mathrm{He}$ and ${ }^{7} \mathrm{Li}$ alone (assuming $N_{\nu}=3$ ), and then use it to set an upper bound to $N_{\nu}$ (as opposed to determining a likelihood function for $N_{\nu}$ ).

The Table illustrates the robustness of the cosmological limit to the number of light particle species. With conservative assumptions about the astrophysical uncertainties the extreme model for ${ }^{3} \mathrm{He}$ chemical evolution and $Y_{P}=0.252$ - less than four massless neutrino species are allowed. A comparable limit follows with $Y_{P}=0.247 \pm 0.003$ and $(\mathrm{D} / \mathrm{H})_{P}<2 \times 10^{-4}$ or no bound to deuterium and $Y_{P}=0.242 \pm 0.003$. Even with the most extreme assumptions - no limit to primeval deuterium and $Y_{P}=0.252 \pm 0.003$ - a meaningful limit still exists, $N_{\nu}<4.9$. Thus, until the current astrophysical uncertainties are clarified we would argue that $N_{\nu}<4$ is a conservative limit, and $N_{\nu}<5$ is a very cautious limit.

Astrophysical and cosmological limits will always have irreducible systematic uncertainty that cannot be quantified with a standard error. In the example at hand, the correctness 
of the standard model of nucleosynthesis must be assumed - though there is no reason to doubt or a compelling alternative - and abundances in the contemporary Universe must be extrapolated to their primeval values. The primary concerns that motivated our reexamination of the big-bang neutrino limit are the systematic uncertainties in the primeval D and ${ }^{4} \mathrm{He}$ abundances, with the former dominated at this time by the chemical evolution of ${ }^{3} \mathrm{He}$. We have shown that these concerns do not preclude setting a meaningful and robust limit to $N_{\nu}$. With conservative assumptions, consideration of ${ }^{7} \mathrm{Li}$ and ${ }^{4} \mathrm{He}$ alone provides the limit, $N_{\nu}<4$. Even with extreme assumptions, the limit $N_{\nu}<5$ follows. The uncertainty due to the chemical evolution of ${ }^{3} \mathrm{He}$ will vanish when the primeval abundance of $\mathrm{D}$ is determined unambiguously in high-redshift hydrogen clouds, which, as we have shown, will sharpen the cosmological limit to the number of light particle species.

Acknowledgments. We acknowledge useful discussions with M. Lemoine, J. Truran, B. Fields and K. Olive. This work was supported by the DoE (at Chicago and Fermilab) and by the NASA (at Fermilab by grant NAG 5-2788 and at Chicago by a GSRP fellowship).

\section{References}

[1] C.J. Copi, D.N. Schramm, and M.S. Turner, Science 267, 192 (1995).

[2] See e.g., P. J. Kernan and L. M. Krauss, Phys. Lett. B 72, 3309 (1994); T. P. Walker, G. Steigman, D. N. Schramm, K. A. Olive, and H. S. Kang, Astrophys. J. 376, 51 (1991).

[3] G. Steigman, D. N. Schramm, and J. E. Gunn, Phys. Lett. B 66, 202 (1977).

[4] J. Yang, M. S. Turner, G. Steigman, D. N. Schramm, and K. A. Olive, Astrophys. J. 281, 493 (1984).

[5] T.P. Walker et al., Astrophys. J. 376, 51 (1991); L.M. Krauss and P.J. Kernan, Phys. Rev. Lett. 72, 3309 (1994) and astro-ph/9405004; K.A. Olive and G. Steigman, Phys. Lett. B 354, 357 (1995); C.Y. Cardall and G.M. Fuller, astro-ph/9603071; P.J. Kernan and S. Sarkar, astro-ph/9603045; B. Fields et al., astro-ph/9603009.

[6] C.J. Copi, D.N. Schramm, and M.S. Turner, Phys. Rev. Lett. 75, 3981 (1995).

[7] M. Spite et. al., Astron. Astrophys. 141, 56 (1984); J.A. Thorburn, Astrophys. J. 421, 318 (1994).

[8] See e.g., M.H. Pinsonneault, C.P. Deliyannis, and P. Demarque, Astrophys. J. Suppl. 78, 179 (1992); B. Chaboyer and P. Demarque, Astrophys. J. 433, 510 (1994); M. Spite and L. Pasquini, Astron. Astrophys. 290, 217 (1994); A.M. Boesgaard, in Formation of the Galactic Halo ... Inside and Out, eds. H. Morrison and A. Sarajedini (Astronomical 
Society of the Pacific, 1996); M. Lemoine, D.N. Schramm, J.W. Truran and C.J. Copi, in preparation.

[9] K.A. Olive and G. Steigman, Astrophys. J. Suppl. 97, 49 (1995).

[10] D. Sasselov and D.S. Goldwirth, Astrophys. J., in press (1995); E.D. Skillman, R. Terlevich, and D.R. Garnett, ibid 411, 655 (1993); ibid 431, 172 (1994).

[11] Y.I. Izotov, T.X. Thuan, and V.A. Lipovetsky, Astrophys. J. (Suppl.), in press (1996).

[12] J.L. Linsky et al., Astrophys. J. 402, 694 (1993); J. Geiss and H. Reeves, Astron. Astrophys. 18, 126 (1972).

[13] H. Reeves, J. Audouze, W.A. Fowler, and D.N. Schramm, Astrophys. J. 179, 909 (1973).

[14] F. T. Adams, Astron. Astrophys. 50, 461 (1976).

[15] D.N. Schramm and M.S. Turner, Nature 381, 193 (1996).

[16] A. Songaila et al., Nature 368, 599 (1994); R.F. Carswell et al., Mon. Not. R. astr. Soc. 268, L1 (1994); M. Rugers and C.J. Hogan, Astrophys. J. 459, L1 (1996); D. Tytler, X.-M. Fan and S. Burles, Nature 381, 207 (1996); S. Burles and D. Tytler, astroph/9603070.

[17] E.J. Wampler et al., Astron. Astrophys., in press (1996) (astro-ph/9512084); R.F. Carswell et al., Mon. Not. R. astron. Soc. 278, 506 (1996); M. Rugers and C.J. Hogan, astro-ph/9603084); L. Cowie and A. Songaila, in preparation (1996).

[18] I. Iben and J. W. Truran, Astrophys. J. 220, 980 (1978); E. Vangioni-Flam, K. A. Olive, and N. Prantzos, Astrophys. J. 427, 618 (1994).

[19] C. Charbonnel, Astrophys. J. 453, L41 (1995); C.J. Hogan, ibid 441, L17 (1995); G.J. Wasserburg, A.I. Boothroyd, and I.-J. Sackmann, ibid 447, L37 (1995).

[20] C.J. Copi, D.N. Schramm, and M.S. Turner, Astrophys. J. 455, L95 (1995).

[21] G. Gloeckler and J. Geiss, Nature 381, 210 (1996).

[22] M.S. Turner et al., Astrophys. J. Lett. 466, L1 (1996).

[23] B. Fields and K.A. Olive, astro-ph/9508344. 


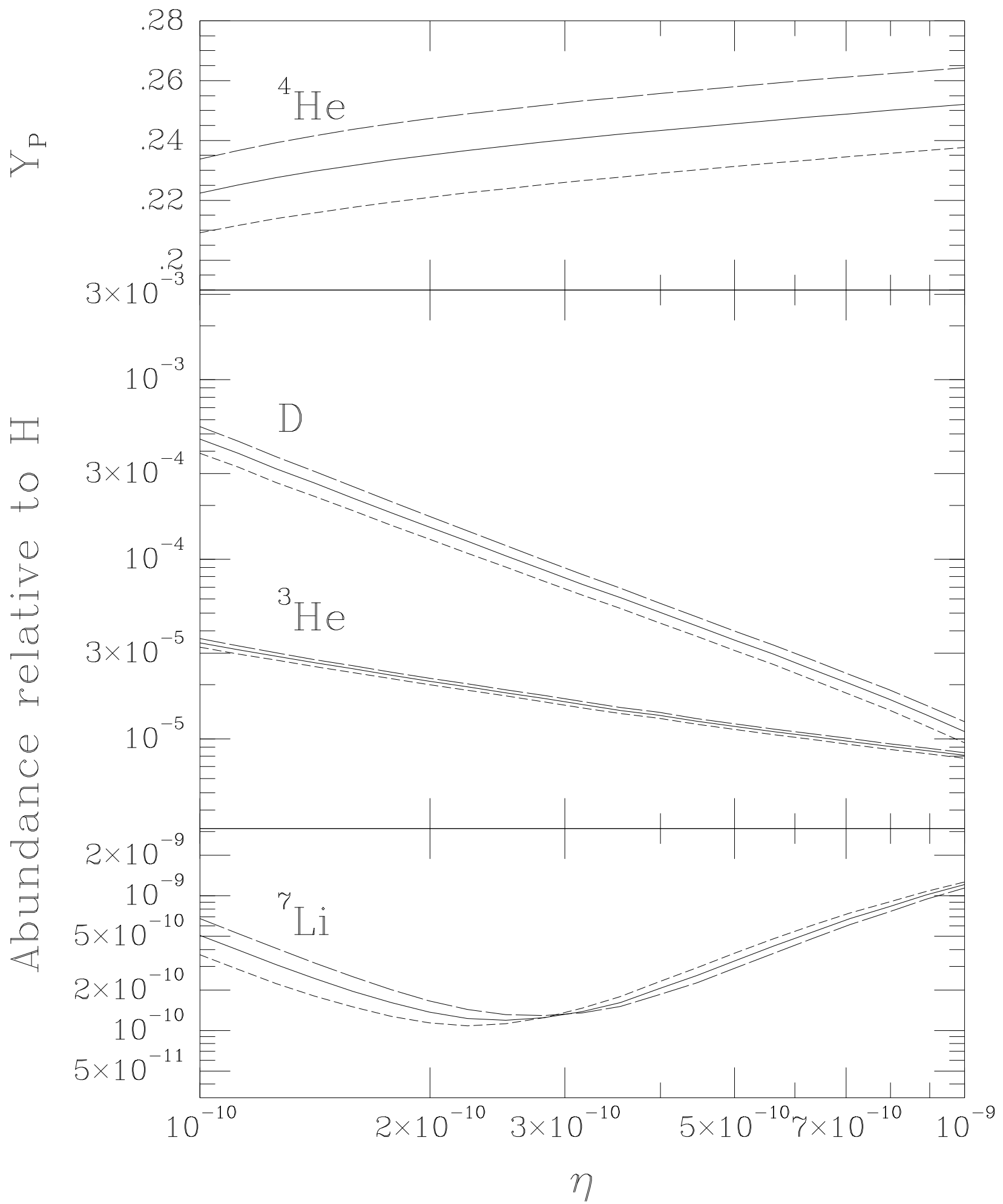

Figure 1: Big-bang production of the light elements for $N_{\nu}=2$ (short-dashed line), $N_{\nu}=3$ (solid line), and $N_{\nu}=4$ (long-dashed line). Note that for low baryon density the yields of both ${ }^{7} \mathrm{Li}$ and ${ }^{4} \mathrm{He}$ increase with $N_{\nu}$ so that ${ }^{7} \mathrm{Li}$ and ${ }^{4} \mathrm{He}$ can be used together to limit $N_{\nu}$. (For clarity, the theoretical uncertainty in the light-element production is not shown; see e.g., Ref. [四.) 


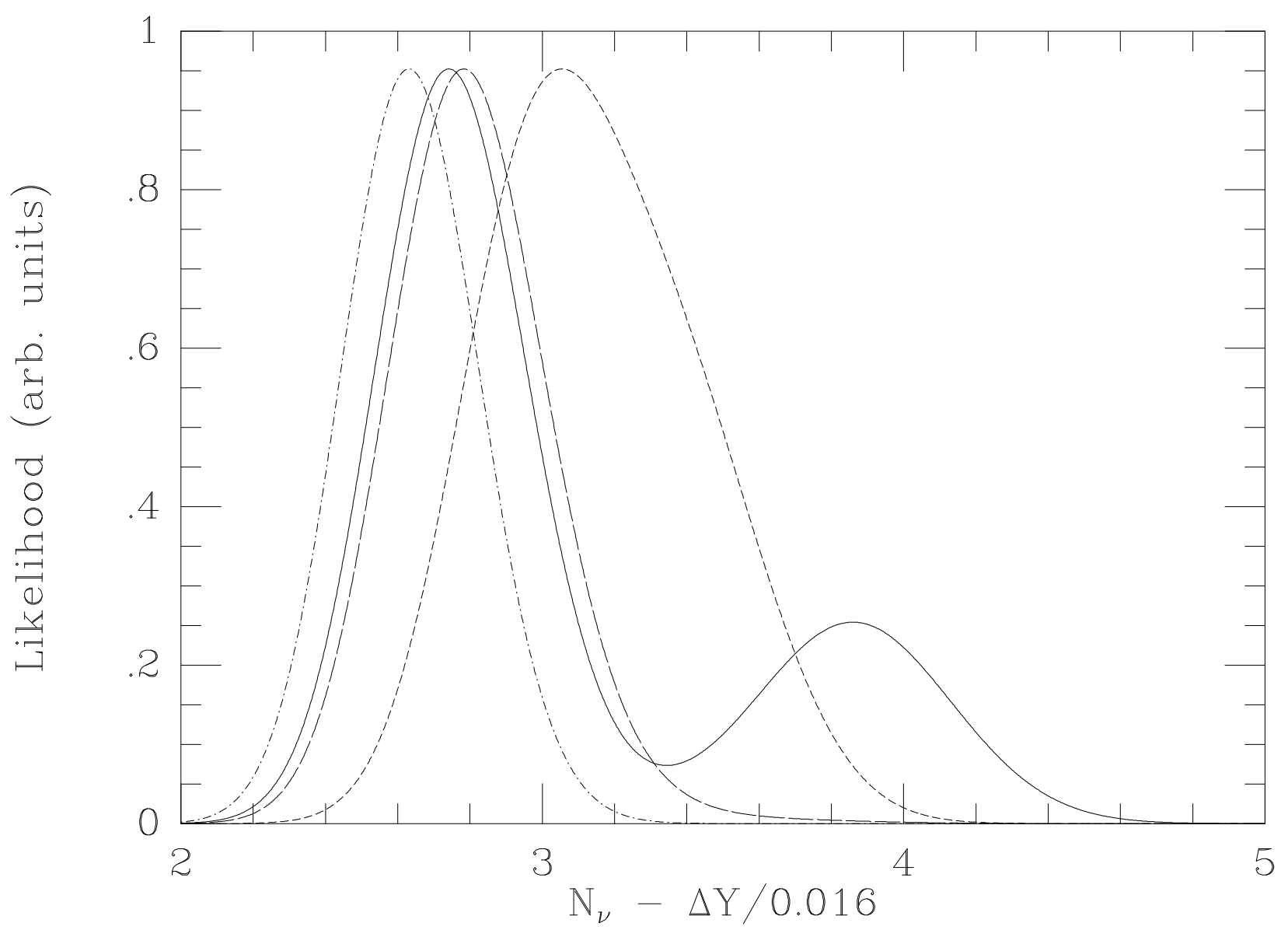

Figure 2: Marginal likelihood for $\tilde{N} \equiv N_{\nu}-\Delta Y / 0.016$ with different Bayesian priors for the primeval deuterium abundance: $(\mathrm{D} / \mathrm{H})_{P} \leq 1.0$ (solid line); $\left[\left(\mathrm{D}+{ }^{3} \mathrm{He}\right) / \mathrm{H}\right]_{P} \leq 2 \times 10^{-4}$ (short-dashed line); extreme model of ${ }^{3} \mathrm{He}$ chemical evolution (from Ref. [22]) (long-dashed line); $(\mathrm{D} / \mathrm{H})_{P}=(2.5 \pm 0.5) \times 10^{-5}$ (dashed-dotted line). In each case we have assumed the ${ }^{7} \mathrm{Li}$ abundance that results in the least stringent limit to $\tilde{N}$. The fact that $\tilde{N}=3$ is well within the $95 \%$ credibility interval is indicative of the consistency of big-bang nucleosynthesis with three massless neutrino species. 